\title{
Improving the Efficiency of Induction Heating in the Air Gap of the Magnetic Circuit
}

Vladimir L. Lanin, Evgeniy Ratnikau, Alexander D. Hatskevich

Electronic Technique and Technology Department, Belarus State University of Informatics and Radioelectronics, Minsk, Belarus

Abstract: Improving the efficiency of induction heating of parts in the air gap of the magnetic circuit is associated with the use of surface and edge effects. Through modeling in ANSYS Electromagnetics Suite 19.2 and experimental studies identified patterns of edge effect in the heated parts. To ensure the uniformity of induction heating of small parts and reduce the soldering time, the electrical switch of soldered parts is used, which with the help of device controller forms a secondary circuit with low electrical resistance and high density of eddy currents.

Keywords: Induction heating; The gap of the magnetic circuit; Edge effect; Secondary circuit

Publication date: July, 2020

Publication online: 31 July, 2020

*Corresponding author: Vladimir Lanin, vlanin@ bsuir.by

\section{Introduction}

Induction heating of parts by eddy currents is widely used in mass production due to high speed, localization of the selective heating zone, the use of any gaseous medium (vacuum, protective gas, air), automation process ${ }^{[1]}$. The choice of the form of the solenoid and magnetic circuit depends on the geometrical dimensions of the heated parts, as well as on their physical properties. Solenoid-type inductors are characterized by significant scattering of the magnetic flux, low efficiency $(0.2-0.4)$, the need for electrical and thermal insulation from the heated surfaces of parts and water cooling during operation. HF energy sources-10-50 kW generators have significant weight and size characteristics and also require forced air or water cooling ${ }^{[2]}$. Solder balls on bonding pads form also induction heating in an electromagnetic field ring inductor $^{[3]}$. The flashing off of solder at the expense of selectiv induction heating allows to avoid the negative effects inherent in traditional methods. Before an induction flashing off balls of solder $\mathrm{Sn} 3,5 \mathrm{Ag}$ in diameter of $0,76 \mathrm{~mm}$ are fixed on bonding pads of a pay from glass-cloth-base laminate FR4 in the thickness of $0,4 \mathrm{~mm}$.

A new direction in the technique of induction heating is the use of a magnetic core with an air gap in which the heated part is placed (Figure 1, b). This method of implementing induction heating has all the advantages of the first, but is devoid of its disadvantages. The essential difference lies in the presence of a magnetic core (ferrite magnetic core), which allows the electromagnetic field of the inductor to be concentrated in the working gap, where the parts are heated. The working winding covers the magnetic core and is connected to the HF generator. The bias winding serves to control the heating process. Changing the bias current, you can change the magnetic permeability of the magnetic circuit, and, consequently, the intensity of the HF field in the gap of the magnetic circuit ${ }^{[4]}$.

The use of a magnetic circuit for the concentration of electromagnetic energy in the region of the gap allows increasing the efficiency of heating due to two zones of surface heating. Increases the speed and localization of heating, which is essential when soldering products of integrated electronics. However, during induction heating, a «edge effect» is observed in the gap of the magnetic circuit, which manifests itself in the distortion of the electromagnetic field and the distribution of heat sources at the edges of the heated body. The edge effect largely determines the quality of heating and the energy characteristics of induction heating devices.

\section{Optimization Inductions with Magnetic}




\section{Core}

\subsection{Modeling}

The most commonly used solenoid (ring) inductors with and without a magnetic core (Figure 1, a). The alternating magnetic flux flowing through the magnetic core causes eddy currents in the conductive material. Vortex electric currents flowing in closed circuits located in planes perpendicular to the direction of the magnetic flux, create their own magnetic flux, tending, in accordance with the Lenz rule, to weaken the change in the main flux. Therefore, they act in a demagnetizing manner, reducing the main flux. The demagnetizing effect of eddy currents varies in different parts of the part. It is most pronounced in the center of the section, since the central parts are covered by the maximum number of current loops. Therefore, in the center of the cross section, the density of the main magnetic flux will be less than at the edges, i.e. there is a displacement of the main magnetic flux in the outer layers of the part. This phenomenon is more pronounced, the higher the frequency of the magnetic flux and the larger the cross section, the magnetic permeability and the conductivity of the material of the part ${ }^{[5]}$.
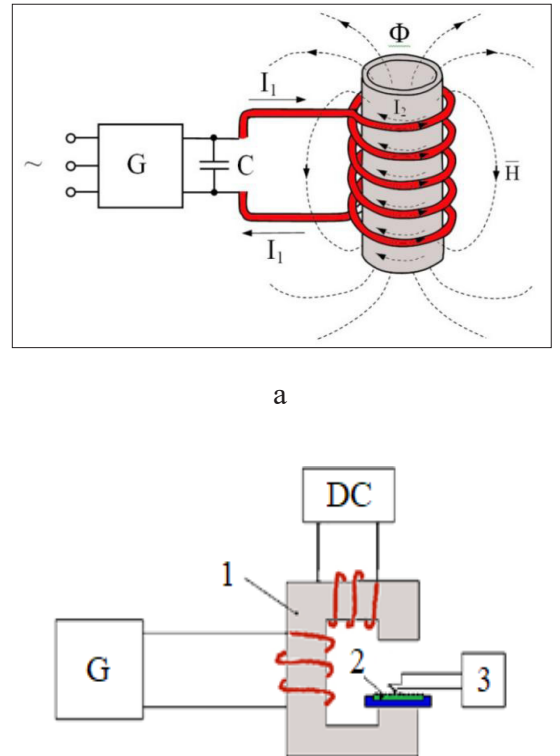

b

Figure 1. Schemes of induction heating by a solenoid inductor (a) and in the air gap of the magnetic circuit (b): 1 - magnetic core, 2 parts, 3 - temperature meter, DC - current source

Simulation of the electromagnetic field in the gap of the magnetic circuit in COMSOL Multiphysics for induction heating of parts from copper alloys at a frequency of $66 \mathrm{kHz}$ and a current of $10 \mathrm{~A}$ showed that there is a non-linear dependence of the depth of heating on the size of the gap (Figure 2). It is noted that $90 \%$ of the electromagnetic field energy is released at a distance of 2-3 $\mathrm{mm}$ from the edge of the part. With an increase in the gap, the heating power falls, but at the same time the heating in the remaining part volume increases ${ }^{[6]}$.

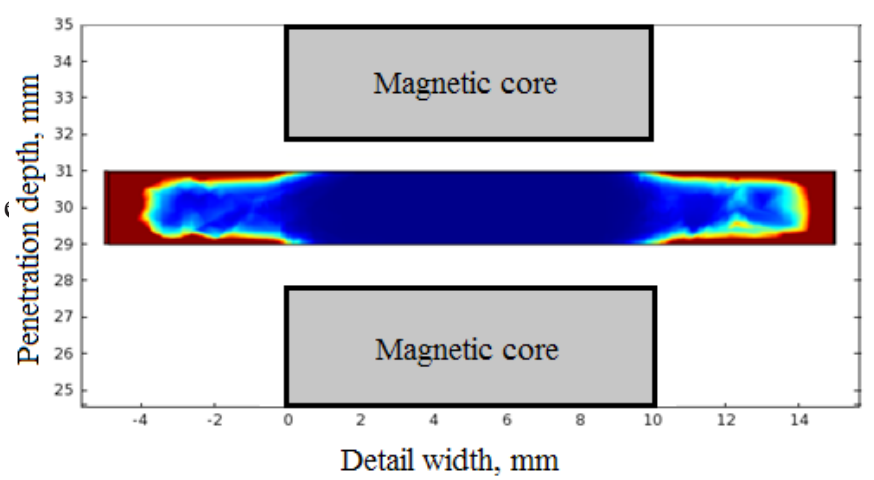

Figure 2. Distribution of specific heating power $(\mathrm{W} / \mathrm{m} 3)$ in depth and along the part

Simulation of the density distribution of eddy currents in parts was carried out in ANSYS Electromagnetics Suite 19.2 using geometric models of induction heating devices on the magnetic core. As a result of modeling, uneven distribution of eddy currents in a metal part was established (Figure 3). The maximum absolute current density (up to $5,6 \cdot 109 \mathrm{~A} / \mathrm{m} 2$ ) at $66 \mathrm{kHz}$ is noted at the edges of the part, and the minimum $(8,6 \cdot 106 \mathrm{~A} / \mathrm{m} 2)$ is at the center of the magnetic core gap.
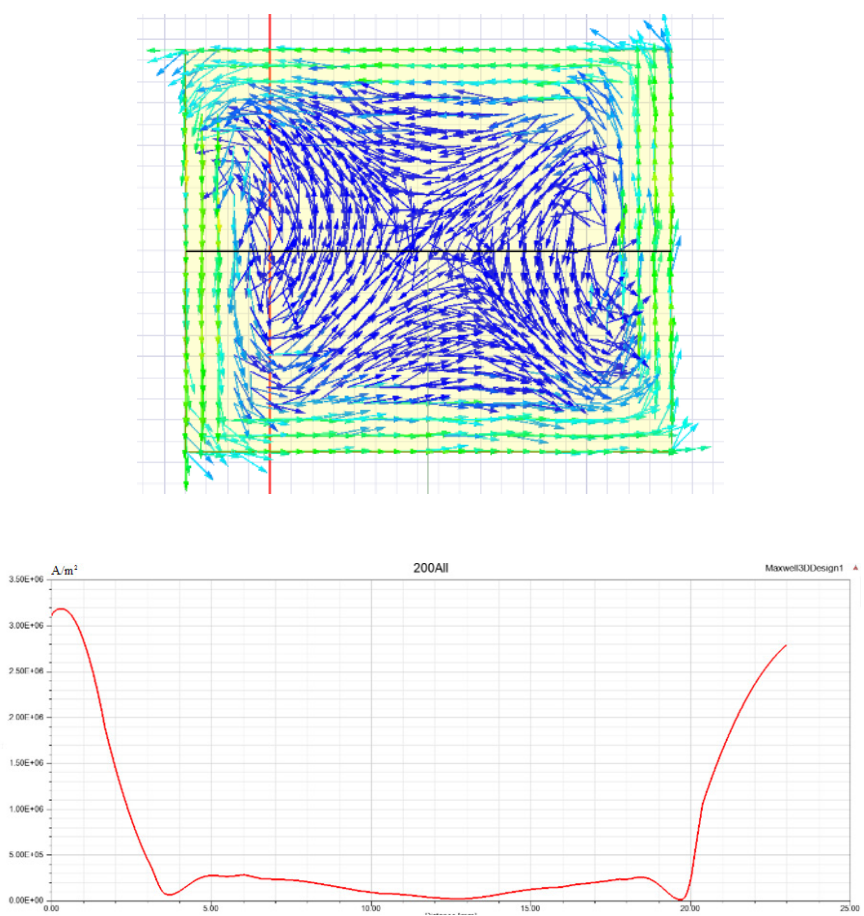

Figure 3. Distribution of current density vectors and current density graph in parts 


\subsection{Experimental}

An induction device for the formation of contact joints with low-melting solders contained a U-shaped magnetic circuit made from ferrite $600 \mathrm{NN}$ with an open magnetic circuit and two windings. Induction winding connected to the HF generator. The parameters of the HF voltage on the working winding were monitored with a voltmeter and frequency meter. The temperature in the working area was measured using a thermocouple $\mathrm{HC}$ attached to the part and a digital temperature meter TRM-205. The magnetic field strength in the gap of the magnetic circuit was estimated by the value of the voltage in the measuring frame. To change the magnetic permeability of the core, a 1-5 A direct current was applied to the additional winding.

The magnitude of the magnetic field was calculated as $^{[7]}$ :

$$
\mathrm{H}=\mathrm{Um} / \mu 0(4,44 \mathrm{~S} \cdot \mathrm{f} \cdot \mathrm{W}),
$$

where Um - voltage amplitude on the frame, $\mu 0$ - absolute magnetic permeability, $S$ - frame area, $f$ - current frequency, $\mathrm{w}-$ number of frame turns.

To improve the efficiency of induction heating of parts in air gap of inductor magnetic circuit, an electric switch of eddy currents, made in the form of a plate made of conductive material, which by means of a regulator device forms a secondary circuit with low electrical resistance and high current density, is used to reduce soldering time ${ }^{[8]}$. This made it possible to regulate the density of eddy currents flowing through the parts, to increase concentration of currents in the heating zone and thereby efficiency of induction heating.

\subsection{Results and discussion}

As a result of the edge effect at the edges of the part subjected to induction heating in the air gap of the magnetic circuit, potential difference arises, which depends on the heating power and on the resistivity of the material of the part. At a heating frequency of 66 $\mathrm{kHz}$, the magnetic field strength was $4.5 \cdot 105 \mathrm{~A} / \mathrm{m} 2$, and the field heterogeneity was $1,3-1,4$ relative to the center of the part (Figure 4).

An induction device on a magnetic core made from ferrite of the brand $600 \mathrm{NN}$, powered by a generator with a frequency of $66 \mathrm{kHz}$ and a power of $1,5 \mathrm{~kW}^{[9]}$, was used to solder the power contacts of the electronic module using solder paste $\mathrm{Sn}-\mathrm{Pb}$. In the resulting secondary circuit formed of soldered contacts and an electric contactor, the value of eddy currents was regulated by the device within 6-10 A. The speed of induction heating increased 2 times and amounted to $50^{\circ} \mathrm{C} / \mathrm{sec}$. With such a high heating rate, effective control of the temperature profile of the soldering of electronic components is necessary ${ }^{[10]}$.

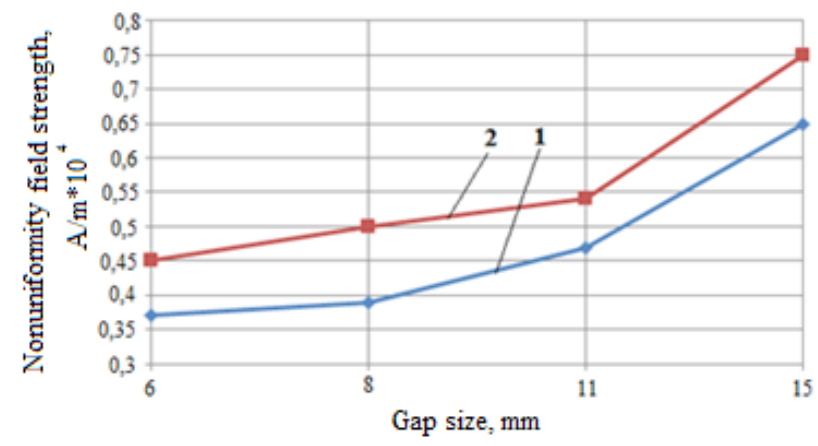

Figure 4. The dependence of the change in intensity relative to the center of the size of the gap at frequencies: $1-66 \mathrm{kHz}, 2-22 \mathrm{kHz}$

\section{Conclusions}

Taking into account the edge effect of induction heating when heating parts in the air gap of the magnetic circuit allows you to more accurately determine the optimal temperature profile of heating and thereby ensure uniform heating of small parts in the process of soldering or heat treatment.

Improving the efficiency of induction heating is achieved through the formation of soldered parts and a secondary circuit controller with a low electrical resistance using an electric contactor, in which an increased density of eddy currents is created, which allows increasing the speed and uniformity of heating of the soldered parts and thereby ensuring high quality of solder joints.

\section{References}

[1] Induction Heating. Industrial Applications / Ed. by S. Lupi. Paris, U.I.E., 1992.

[2] Rudnev V. Handbook of Induction Heating. N. Y.: Marcel Dekker, 2010.

[3] Xu H., Li M., Fu Y., Wang L., Kim J. Local Melt Process of Solder Bumping by Induction Heating Reflow. Soldering @ Surface Mount Technology, 2009, № 4, 45-54. DOI. 10.1109/ TADVP.2008.923385

[4] Lanin V.L. Local High-Frequency Heating in Electronics Technology. Journal of Electronic Research and Application, 2019. V. 3, № 4, 1-6. DOI: 10.26689/jera.v3i4.824.

[5] Lanin V.L. Application of the Concentrated Power Streams in Electronics Industry. Saarbrucken, Germany: Scholar's Press. 2015.

[6] Marvin D. How Induction Soldering Improves The Process and Quality of Coax and Interconnect Assembly. 2014. iTherm 
Technologies www.ithem.com

[7] Nemkov VS, Demidovich VB. Theory and calculation of induction heating devices. St. Petersburg: Energoatomizdat, 1988.

[8] Lanin VL. High-Frequency Heating for Soldering in Electronics. Circuits and Systems, 2012, № 3, 238-241. DOI 10.4236/cs
2012.33033.

[9] Lanin V. L., Sergachev I.I. Induction Devices for Assembly Soldering in Electronics. Surf. Eng. Appl. Electrochem., 2012, № 4, 384-388. DOI: $10.3103 / \mathrm{S} 1068375512040114$

[10] Rapoport E., Pleshivtseva Y. Optimal Control of Induction Heating Processes. NY: CRC Press, 2007. 